\title{
Correlation between Wear Resistance and Lifetime of Electrical Contacts
}

\author{
Jian Song, Christian Koch, and Liangliang Wang \\ Precision Engineering Laboratory, Ostwestfalen-Lippe University of Applied Sciences, Liebigstraße 87, 32657 Lemgo, Germany \\ Correspondence should be addressed to Jian Song, jian.song@hs-owl.de \\ Received 13 December 2011; Accepted 6 February 2012 \\ Academic Editor: Dae Eun Kim \\ Copyright () 2012 Jian Song et al. This is an open access article distributed under the Creative Commons Attribution License, \\ which permits unrestricted use, distribution, and reproduction in any medium, provided the original work is properly cited.
}

Electrical contacts are usually plated in order to prevent corrosion. Platings of detachable electrical contacts experience wear because of the motion between contacts. Once the protecting platings have been worn out, electrical contacts will fail rapidly due to corrosion or fretting corrosion. Therefore the wear resistance of the platings is a very important parameter for the long lifetime of electrical contacts. Many measures which improve the wear resistance can diminish the conductivity of the platings. Due to the fact that platings of electrical contacts must have both a high wear resistance and a high electrical conductivity, the manufacturing of high performance platings of electrical contacts poses a great challenge. Our study shows firstly the correlation between the wear resistance of platings and lifetime of electrical contacts and then the measures, which improve the wear resistance without impairing the electrical performance of the contacts.

\section{Introduction}

Copper and copper alloys are the most widely used base materials for electrical contacts. Both copper and copper alloys are prone to corrosion and oxidation. Therefore electrical contacts are usually coated with protecting platings. The most extensively used coating materials are tin, silver, gold, palladium, nickel, and diverse alloys of these metals. Fretting is considered to be another major problem that affects the lifetime of electrical connectors. Different phenomena are related to fretting, such as fretting wear, fretting fatigue, fretting corrosion, insulating layer, and oxides [1-3]. Among these phenomena corrosion, insulating layer, and oxides are basically the direct cause of a major increase in contact resistance. The platings of nonprecious metals are generally prone to fretting corrosion. Therefore using metals which have little or no film-forming tendency, generally known as precious metals, as plating materials should be an effective measure to avoid failures caused by fretting. Gold is one of the most commonly used precious plating materials for high performance electrical contacts. Pure gold is very soft. When using gold as coating material, one of the limitations to their lifetime is the wear resistance of the plating material. This has been observed by some authors $[1,2]$. Hard gold is usually used in order to improve the wear resistance of gold platings. The high degree of hardness is achieved by alloying elements such as cobalt, iron, or nickel. However, the effect of alloying elements is limited by the galvanic process and other surface properties, which are also required for electric contacts.

Instead of alloying elements, nanoscale particles, mostly metal oxides, can also be used for the modification of platings. The reason for using nanoscale particles is based on the fact that the hardness of pure gold is about HV 70 and the hardness of hard gold is about HV 170 [3]. The hardness of nanoscale particles of metal oxides ranges from HV 700 to 2300 [4]. It is well known from the study of tribology that in general hard materials are more wear resistant than soft materials, provided other characteristics of the materials are the same [2]. PTFE reduces the friction between contacts and therefore the wear. These can provide an additional lifetime improvement [5]. However PTFE is basically an electrical isolator. Therefore it has to be used with care for electrical contacts in order to alleviate increase of contact resistance.

\section{Experimental}

For the wear and fretting corrosion tests an apparatus which enables a small and precise displacement of fretting motion 


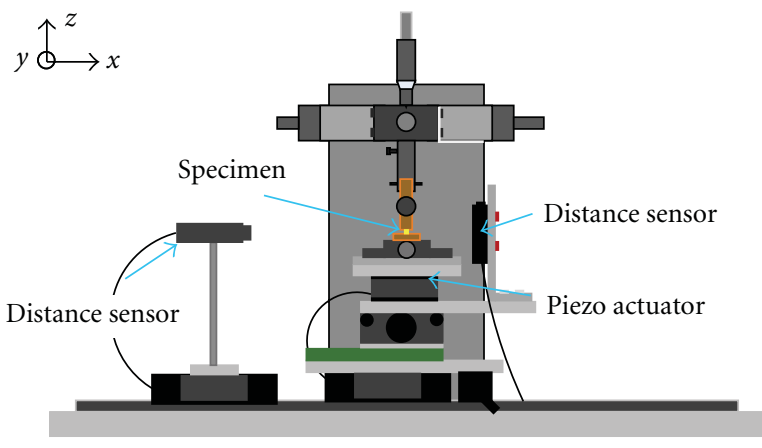

FIGURE 1: Apparatus for wear and fretting corrosion tests.

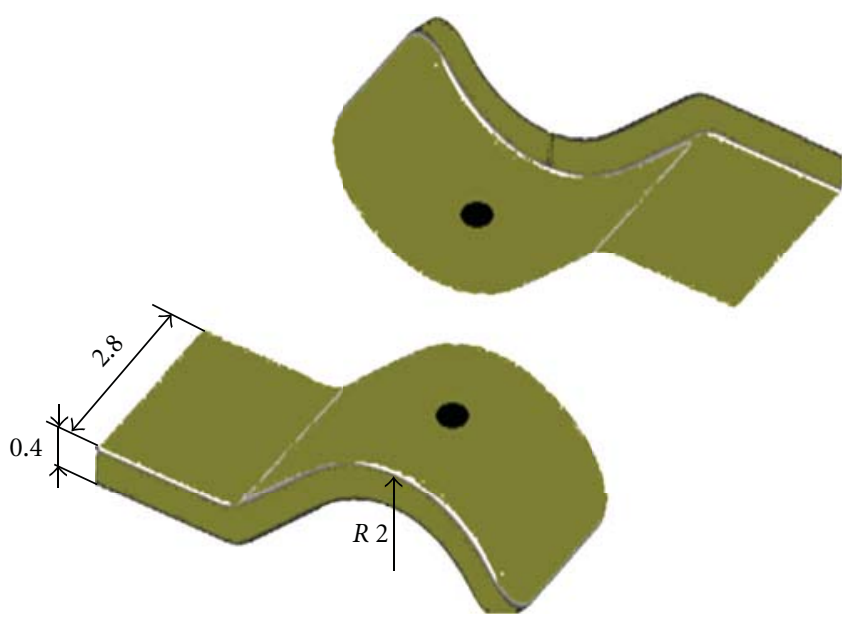

FIgURE 2: Contact springs.

at the contact interface is used (Figure 1). A piezoelectrical actuator moving forwards and backwards generates the relative motion between the contacts. The wipe is $50 \mu \mathrm{m}$, which relates to the displacement caused by the difference in thermal expansion between PCB and connectors. The duration of a cycle is $1 \mathrm{~s}$, which is slow enough to enable the chemisorption of oxygen on the surface and sufficiently fast to enable an acceptable test time. The contact force is provided with a dead load. The normal force is $2 \mathrm{~N}$. The contacts are wired for a four-wire resistance measurement. A computer controls the data acquisition system.

A transmission electron microscope (TEM) combined with an image processing program was used for characterization of nanoscale particles powder. An Ultrafine Particle Analyzer (UPA), which incorporated the Controlled Reference Method (CRM) in a dynamic light scattering instrument, was used to determine the particle size distribution in electrolytes. The wear of the contact area was measured after the wear test with a confocal laser scanning microscope (CLSM), which measured the surface topography with a precision of up to $10 \mathrm{~nm}$. A scanning electron microscope (SEM) with a Focused Ion Beam System (FIB) and an energy-dispersive X-ray spectroscopy (EDX), an X-ray fluorescence spectrometer, and an optical microscope were also used for the material and surface analysis of the platings.

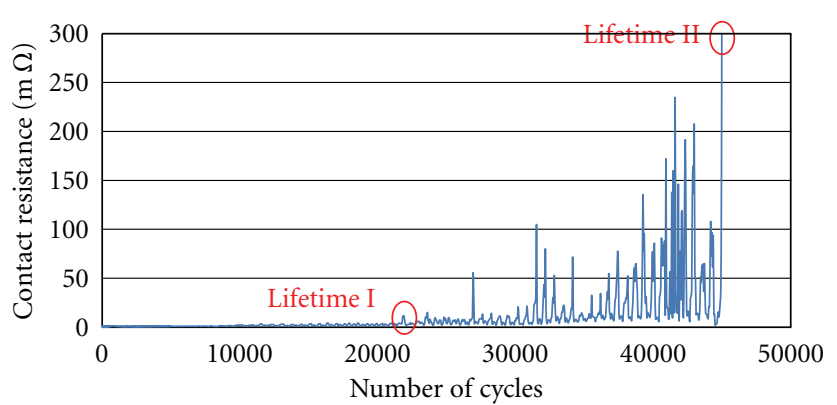

FIGURE 3: Increases in contact resistance due to fretting corrosion [6].

The contact springs were stamped. The base metal used was phosphor bronze (CuSn). The size and setup are shown in Figure 2.

The plate materials tested in this study were tin, silver, pure gold, and hard gold, which were modified with different hard alloying elements, $\mathrm{Fe}, \mathrm{Co}$, or $\mathrm{Ni}$, and nanoscale particles. The thickness of the tin and silver plates was about $3 \mu \mathrm{m}$. The thickness of gold platings is generally much thinner because of the high cost of gold. The thickness of gold platings used for the study was between 0.6 and $1 \mu \mathrm{m}$. In the case of gold platings an interlayer (barrier layer) of nickel was utilized, which prevents the diffusion between gold and base material and building of an intermetallic phase. The materials of nanoscale particles used for the investigation were aluminium or titanium oxides and PTFE. Nanoscale particles of aluminium and titanium oxides were made by IME of RWTH University Aachen. Different peak sizes and size distributions were used for the investigation in order to find out the influence of particle sizes. PTFE was X-7986 of Shamrock Technologies. The PTFE has an average particle size of approximately $200 \mathrm{~nm}$. The samples were all electroplated. The mating parts for each test were always coated with the same material. In the case of modification with nanoscale particles, these were added to electrolytes without pretreatment and stirred by vibration in order to achieve an even distribution of particles in electrolytes. One of the process parameters to influence the concentration of nanoparticles in platings was the amount of nanoscale particles adding to electrolytes. It was expected that with increasing concentration of nanoscale particles in electrolytes more nanoscale particles would find their way to platings.

Several criteria can be used to determine the lifetime of electrical contacts. The number of cycles to the rapid increase of contact resistance was used in this study as lifetime, since it has the strongest impact on the behavior of electrical contacts. Two limits are set for the lifetime. Lifetime I is defined as the number of cycles which leads to a $300 \%$ increase of contact resistance and lifetime II as the number of cycles when the contact resistance of $300 \mathrm{~m} \Omega$ is measured [6]. At the end of lifetime I first damages on platings occur and the contacts can normally still work properly. At the end of lifetime II a normal function of electrical contacts cannot be expected (Figure 3 ). 


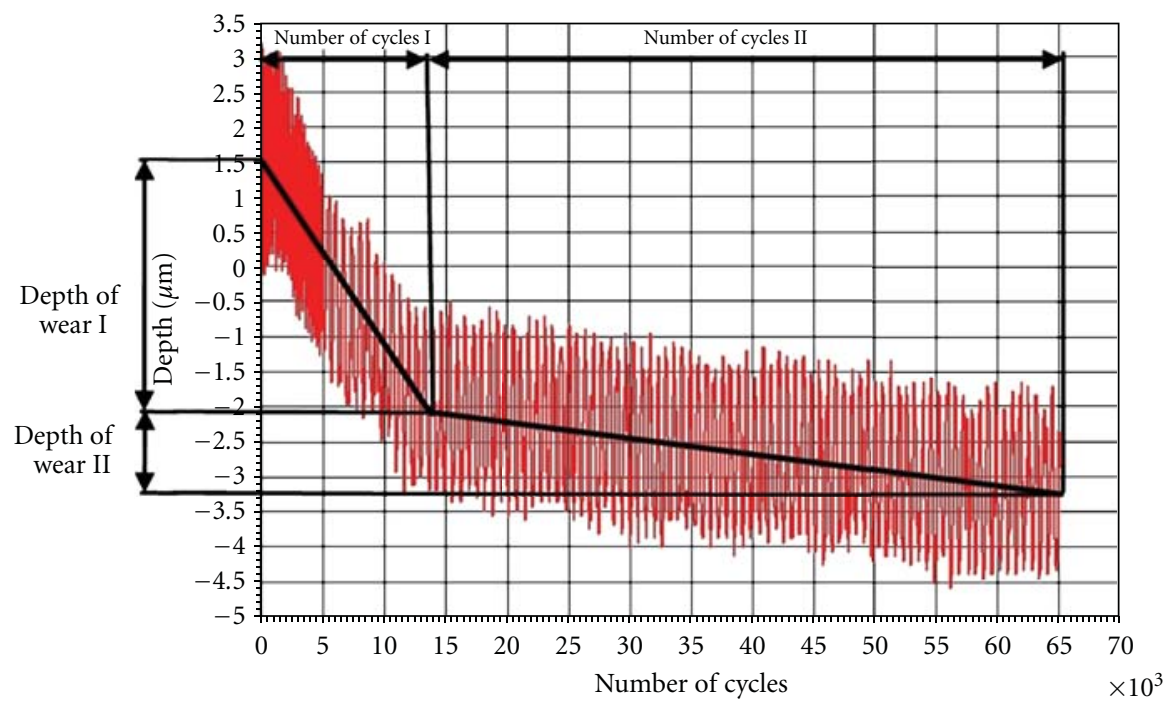

Figure 4: Two phases of wear.

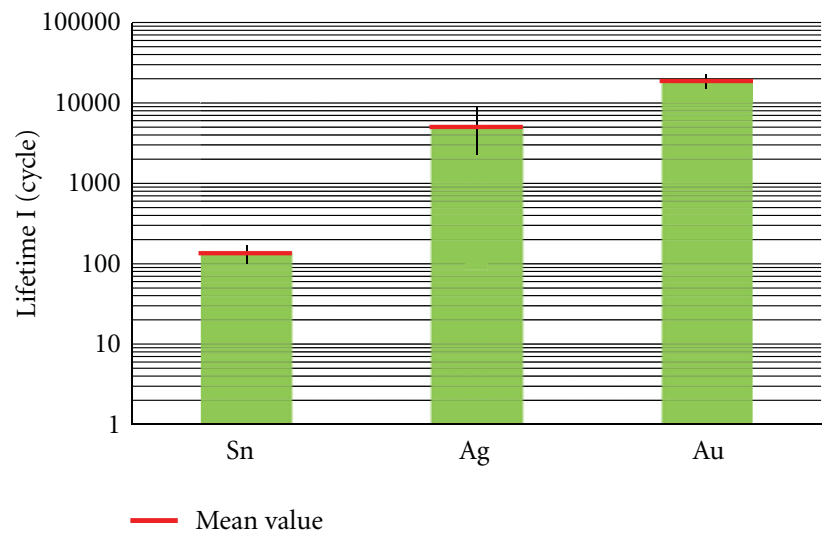

(a)

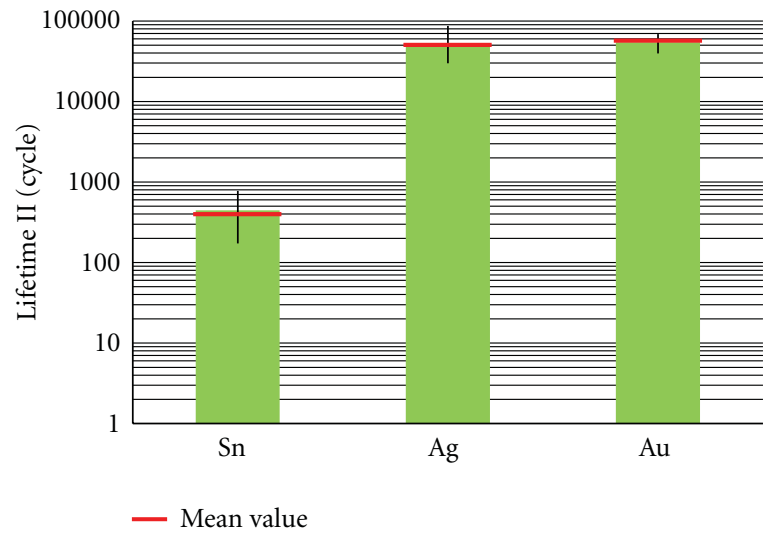

(b)

FIGURE 5: (a) Lifetime I of contacts with different coating materials. (b) Lifetime II of contacts with different coating materials.

The wear of electrical contacts was measured simultaneously to the contact resistance during the tests. The rate of wear, which is defined as depth of wear per 1000 cycles of motion, can be calculated from the wear curve. There are typically two phases of wear. The first phase is the wear of the over plate, which normally takes place faster than the second phase. The second phase is the wear of the interlayer or the base metal (Figure 4). There is usually a very good correlation between phase I of wear and lifetime I of electrical contacts, since the instant of time of phase I of wear corresponds to the instant of time of a partially worn-out plate which leads to first instabilities of electrical contacts. The rate of wear in phase I of wear represents the wear performance of platings. This is therefore relevant for the study of platings.

\section{Results and Discussion}

Figures 5(a) and 5(b) show the lifetimes I and II of electrical contacts with platings of tin, silver, and pure gold (soft gold). Lifetime I of all tin platings was only about 100 cycles. That means that initial damage was observed after only a very short period of operation time. The reason for this was the very soft tin platings, which were partially worn out after a very small number of motion cycles. Contacts with both silver and soft gold platings displayed much longer lifetimes I and II. It should be mentioned at this point that the thickness of silver platings $(3 \mu \mathrm{m})$ was much higher than that of gold platings $(0.6$ and $1 \mu \mathrm{m})$.

The meaning of wear resistance for the lifetime of electrical contacts is obvious when looking at the correlation between the rate of wear and the lifetime of electrical contacts 


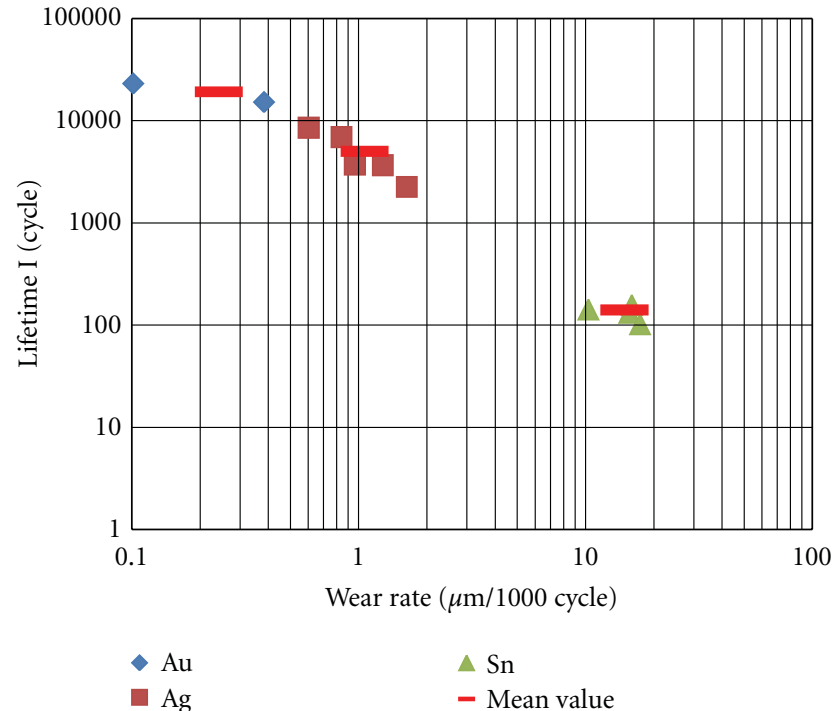

(a)

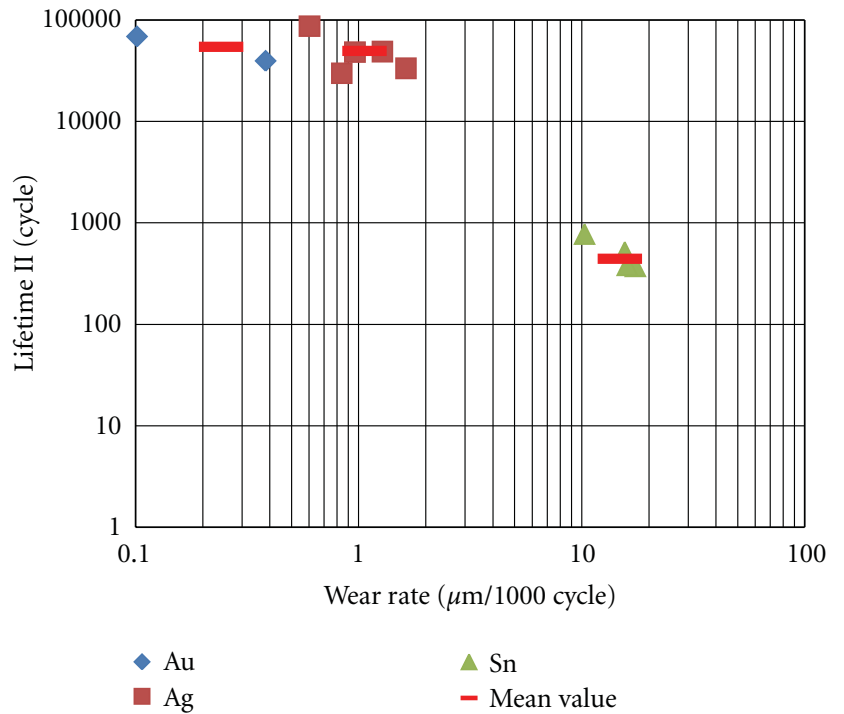

(b)

Figure 6: (a) Correlation between the rate of wear and lifetime I of electrical contacts with different coating materials. (b) Correlation between the rate of wear and lifetime II of electrical contacts with different coating materials.

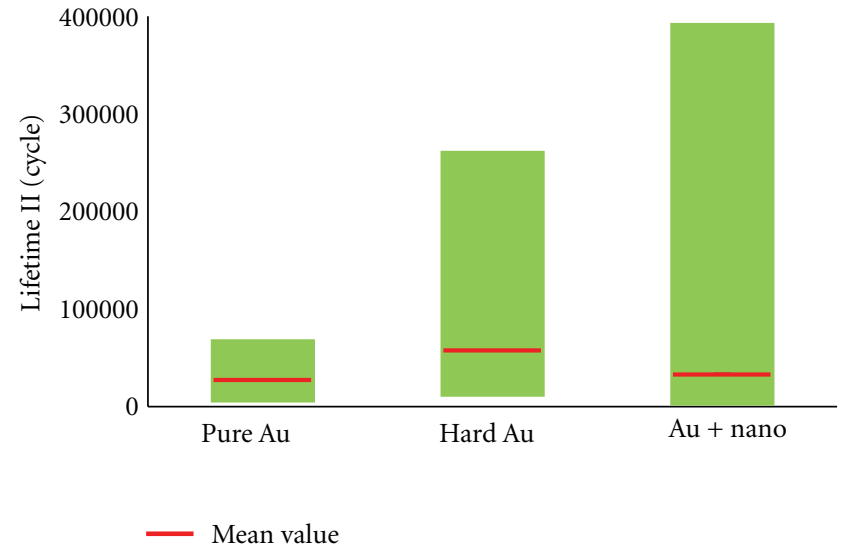

FIGURE 7: Effect of nanoscale particles.

(Figures 6(a) and 6(b)). The best correlation was observed between the rate of wear and lifetime I, since the first instabilities occurred as soon as the first partial wearout of the over plate began. Lifetime II is a practical definition, but physically the definition is somewhat blurred. The degree of electrical resistance increase depends partly on the distribution of debris in the contact zone, especially in case of precious metals. In this phase both wear and transportation of debris of the plate play an important role in the electrical resistance. Different wear patterns can be observed, which will be discussed later in this paper.

The lifetime of gold platings can be further improved by means of modification of gold platings with hard alloying elements or nanoscale particles. The effect of different nanoscale particles on the lifetime of the electrical contacts in the wear and fretting corrosion tests, in comparison to pure gold and hard gold, which is most widely used for high performance electric contacts, is shown in Figure 7. The large range of lifetime is not due to a single runaway value but is verified by numerous measurements. The large lifetime range shows on the one hand the extensive potential of nanoscale particles modified gold platings and on the other hand the large number of parameters in the process chain from generation of nanoscale particles to the nanoscale particles modified gold platings, which yet have to be investigated and characterized.

A very good correlation between the rate of wear of gold platings and the lifetime of electrical contacts in wear and fretting corrosion tests could be observed both for lifetime I and II (cf. Figures 8(a) and 8(b)). With increasing rate of wear (decreasing wear resistance) the lifetime of electrical contacts decreases. The rate of wear of pure (soft) gold is comparable with that of tin. Through the modification of gold, the rate of wear can be decreased by two to three decades and consequently the lifetime of electrical contacts can also be increased by more than two decades. This means that many measures used for improving bearing materials and experience gained in that area can also be applied for coating materials of electrical contacts. It is also to be noted that in terms of coefficient of friction the requirements for both bearing and electrical contacts are similar. A low coefficient of friction leads to a low insertion force for electrical connectors, which is desired.

The fact that there is a very good correlation between the rate of wear and lifetime endorses the approach of the investigation. An increased wear resistance of the platings can lead to a long lifetime of electrical contacts with gold platings. 


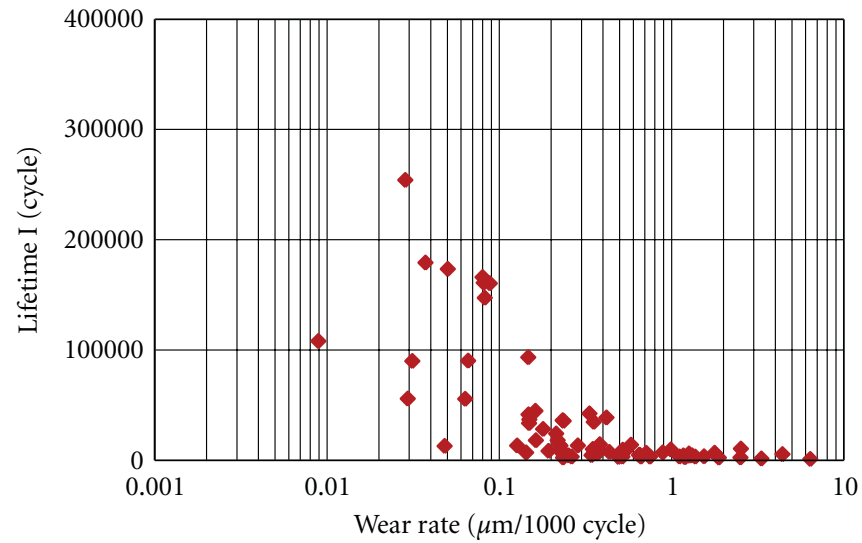

(a)

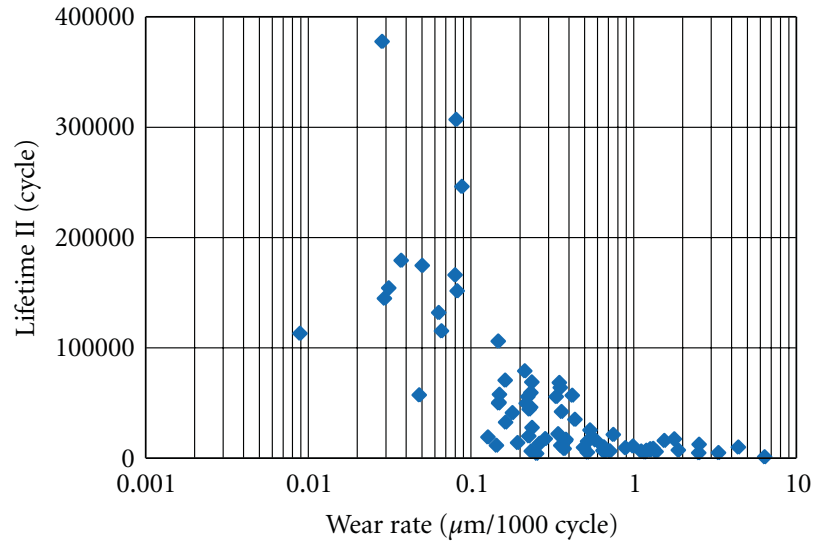

(b)

Figure 8: (a) Correlation between rate of wear of gold platings and lifetime I of electrical contacts. (b) Correlation between rate of wear of gold platings and lifetime II of electrical contacts.

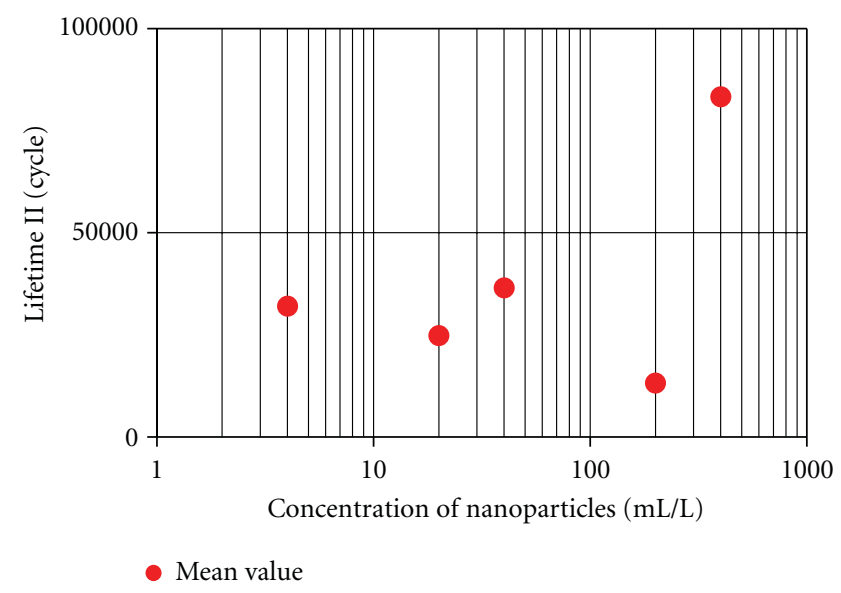

FIGURE 9: Effect of concentration of nanoscale particles in electrolyte (particles in liquid).

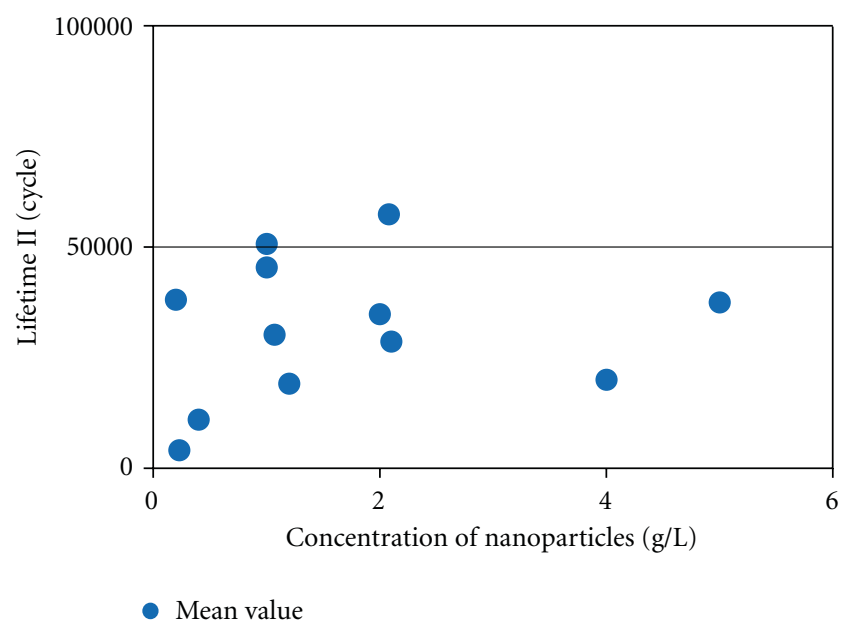

Figure 10: Effect of concentration of nanoscale particles in electrolyte (particles as powder).

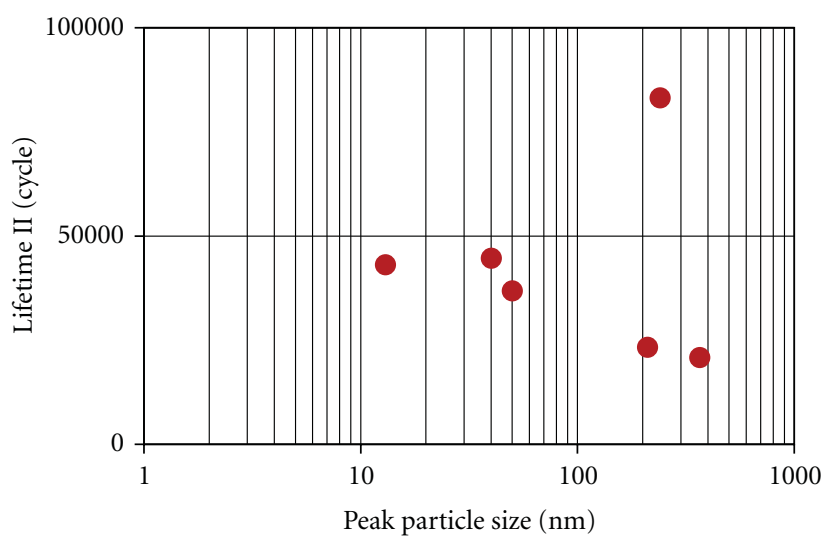

- Mean value

FIgURE 11: Effect of peak particle size in powder.

The fact that the nanoscale particles modified platings show both an extraordinary long and short lifetime reveals that

(i) there is a large potential in terms of improving the lifetime of gold platings with nanoscale particles;

(ii) putting nanoscale particles alone in the platings would not be sufficient. Other basic conditions have to be taken into account in order to achieve the desired effects.

Therefore, further analysis was conducted to study these basic conditions.

3.1. Concentration of Nanoscale Particles in Electrolytes and in Platings. The samples were plated with electrolytes of different nanoscale particle concentration. From a certain concentration of nanoscale particles, samples with long lifetimes were observed (Figure 9). A further increase of the concentration did not improve the performance of the platings 


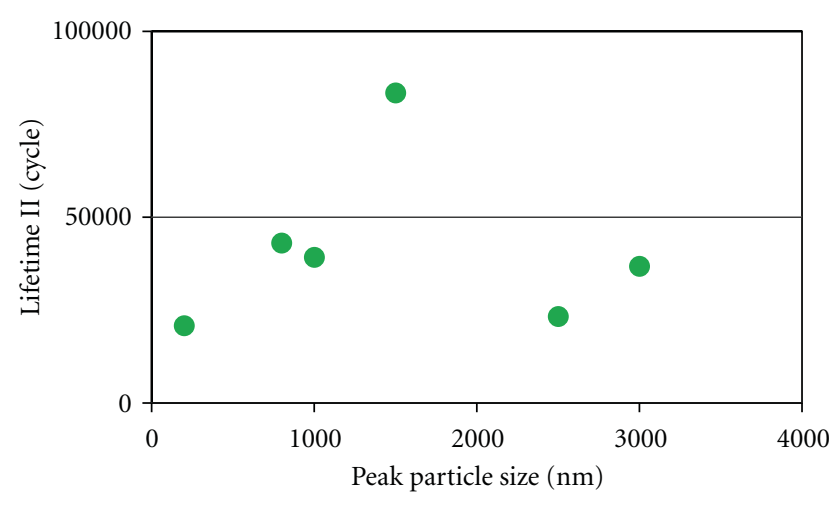

- Mean value

FIGURE 12: Effect of peak particle size in electrolytes.

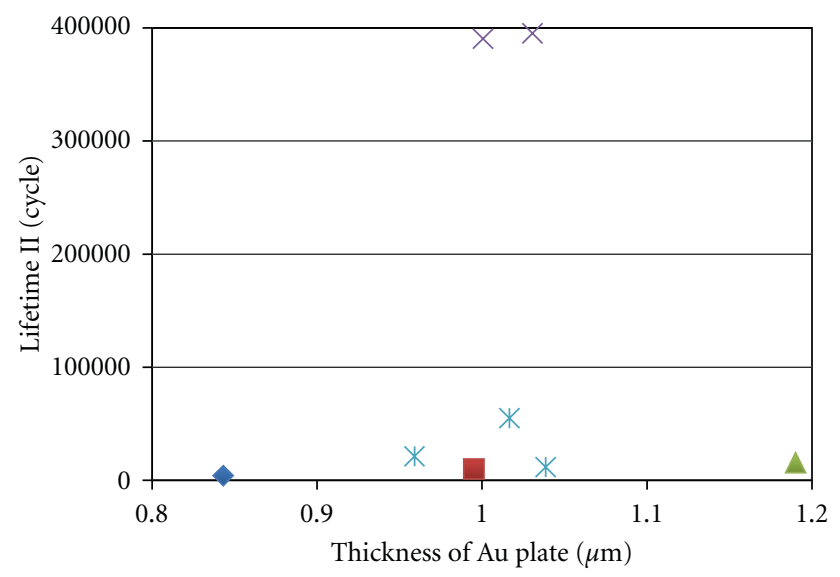

Figure 13: The lifetime and the thickness of gold platings.

(Figure 10). The investigation showed that neither a correlation between the concentration of nanoscale particles in electrolytes and platings, nor a correlation between the lifetime of electrical contacts and the concentration of nanoscale particles in platings existed. These facts mean that the amount of nanoscale particles in platings is not a determining parameter for the lifetime of electrical contacts. Since we know that there is a good correlation between the rate of wear of gold platings and the lifetime of electrical contacts, these results also illustrate that the amount of nanoscale particles in platings is not a determining parameter for the wear resistance.

3.2. Effect of Peak Particle Size. The peak particle size is defined as the most frequently observed size of particles. It is therefore one of the characteristics of particles. Smaller particles are in general more difficult to produce than larger ones, but thought to be more strongly bound to the matrix material because of their larger specific surfaces (surface/volume). Figure 11 shows that this does not necessarily lead to a long lifetime.

Nanoscale particles tend to agglomerate in electrolyte. Due to this the peak particle size measured in electrolytes is much larger than that in powder or water. Figure 12 shows that if the agglomerates become too large, the average

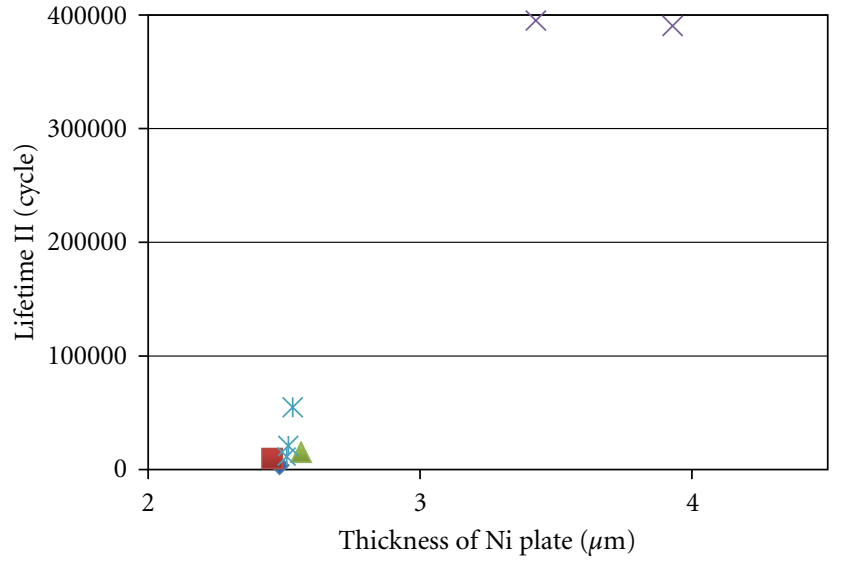

FIGURE 14: Effect of thickness of nickel interlayer.

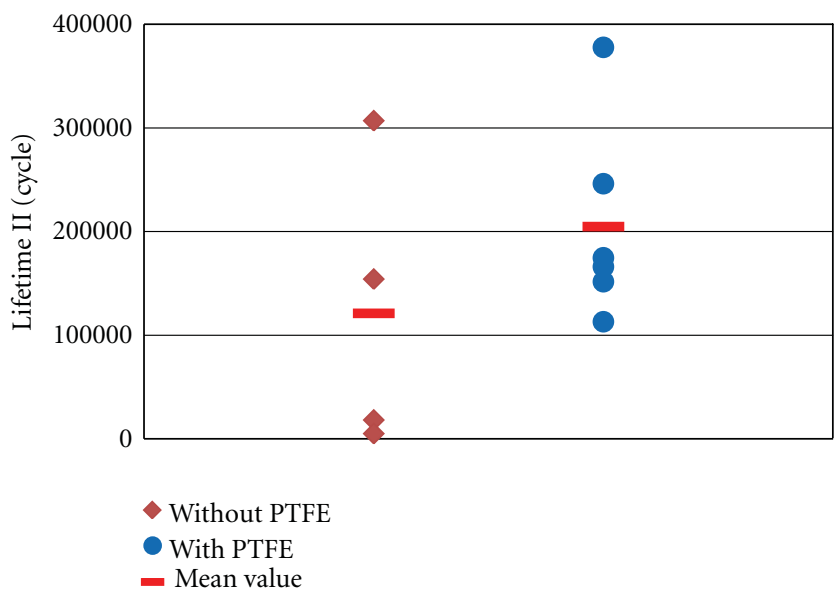

FIGURE 15: Effect of PTFE particles on lifetime.

lifetime will be shortened. The limit seems to be the thickness of the gold plate.

3.3. Effect of the Thickness of Platings. Since the wear of platings is proportional to the cycles of motion, the number of cycles to the wear-out of the gold plate should be proportional to the thickness of the gold plate. Once the gold plate is worn out, the fretting corrosion of the interlayer can commence. Therefore a dependence of lifetime on the thickness is expected. Our results showed that a minimum thickness of approximately $0.7 \mu \mathrm{m}$ is required to achieve a long lifetime. However, thickness is a necessary but not sufficient condition for a long lifetime. Some samples with a thick plate also displayed a short lifetime.

Nickel interlayer have a strong effect on the lifetime of electrical contacts. Samples with nickel interlayer of $2.3 \mu \mathrm{m}$ or less survived less than 10,000 cycles. All samples which survived an exceptionally large number of cycles of more than 200,000 have a nickel interlayer of more than $3 \mu \mathrm{m}$. The detailed analysis of a batch of nanoscale particles, which showed the longest average lifetime of about 120,000 cycles, confirmed the importance of nickel interlayer. At first glance there is also a very large scattering of results. The thickness 


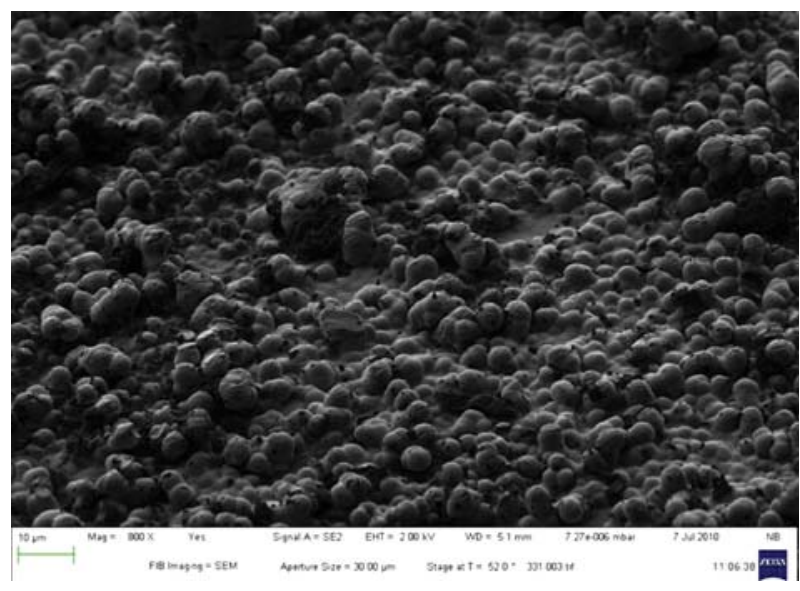

FIGURE 16: Spherical structured surface (lifetime: 400,000 cycles).

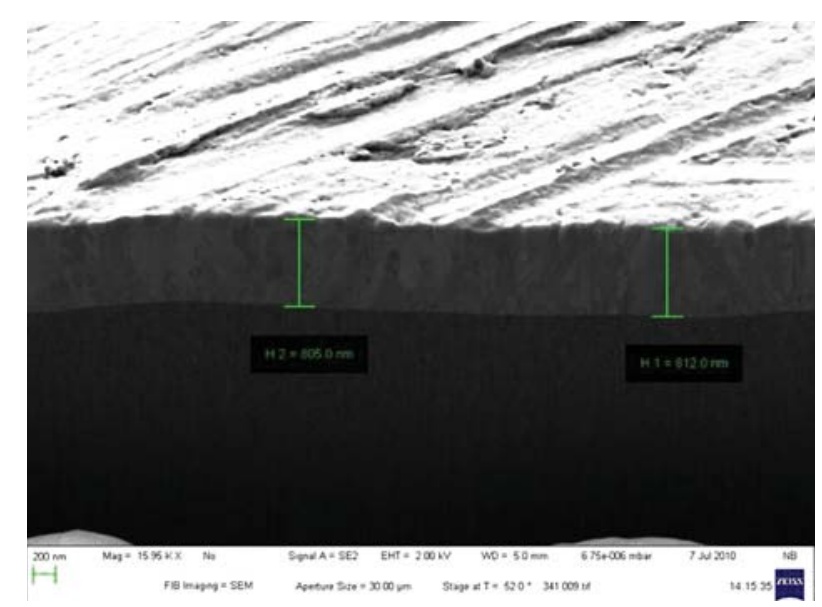

FIgURE 17: Smooth surface (lifetime: 55,000 cycles).

of the batch ranged from 0.85 to $1.2 \mu \mathrm{m}$. However the best samples had a thickness of $1 \mu \mathrm{m}$ (Figure 13). Nevertheless the determining importance of nickel interlayer could be clearly demonstrated; see Figure 14.

A similar phenomenon was also observed for hard gold platings. The reason for this phenomenon is the subsurface plastic deformation, which results in a deepening wear groove on the surface and the formation of buckles in the interlayer. Above these buckles, localized surface wear occurs. With repeated cycling, exposure of interlayer material eventually occurs at these sites. This occurrence is indicative of a local bulging of the interlayer through the final coating. It was found that with sufficiently thick and stiff platings the mechanism could be eliminated [2]. Another reason for this phenomenon is the dependence of the interlayer roughness on the thickness of interlayer. It was found that the interlayer roughness decreased with increasing thickness of up to $4 \mu \mathrm{m}$ [7]. It should be mentioned at this point that the thickness of interlayer is of course not the only determining parameter for the lifetime of electrical contacts with nanoscale particles modified gold platings. The amount of nanoscale particles in gold platings must be above a certain limit, which can be

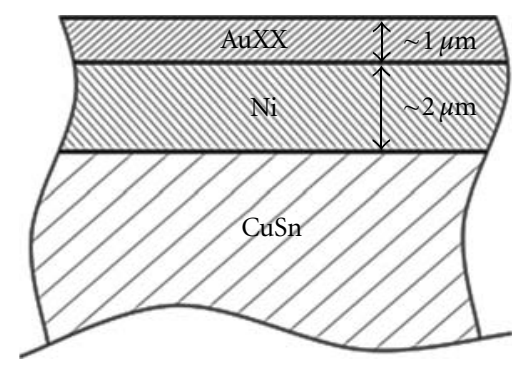

Figure 18: Cross-section of plating system of electric contacts before the wear and fretting corrosion test.

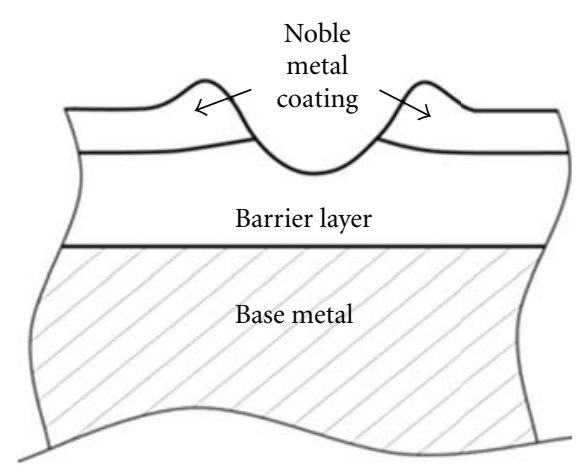

FIGURE 19: Wear pattern In total wearing off of the upper layer. Cross-section of plating system of electric contacts after the wear and fretting corrosion test.

indirectly determined by the concentration of nanoscale particles.

3.4. PTFE Particles. As expected, specimens with PTFE displayed on average a longer lifetime (Figure 15). In order to alleviate the negative effect on electrical contact resistance, the smallest available PTFE particles of $200 \mathrm{~nm}$ were used for the contacts.

3.5. Topography of Gold Platings. Once the comparable amount of nanoparticles is in the gold platings, the surface topography is also an important factor. A spherical structured surface (Figure 16) seemed to be more favorable than a smooth surface (Figure 17). The nanoscale particles used, the thickness of gold platings, and the amount of nanoscale particles in gold platings were identical in both cases. The thickness of gold platings was $1 \mu \mathrm{m}$ and the proportion of nanoscale particles was $1 \%$.

3.6. Wear Patterns. Starting with the plating systems shown in Figure 18 three wear patterns can possibly develop [8].

The first wear pattern is the total wearing off of the upper layer (Figure 19). In this case we have the direct contact of the barrier layers, which is sensitive to fretting corrosion. Therefore the electric contacts fail shortly after this stage has been reached.

The second wear pattern is the most favored one for electric contacts. Precious metal pieces are found in the contact 


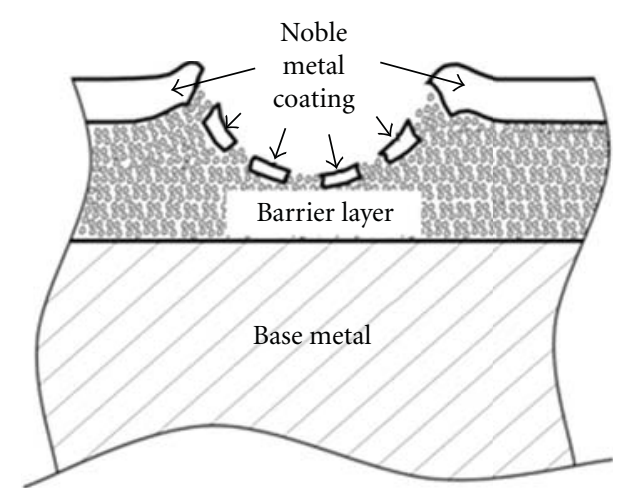

FIGURE 20: Wear pattern II partial wearing off of the upper layer and contact surface not totally covered with oxide.

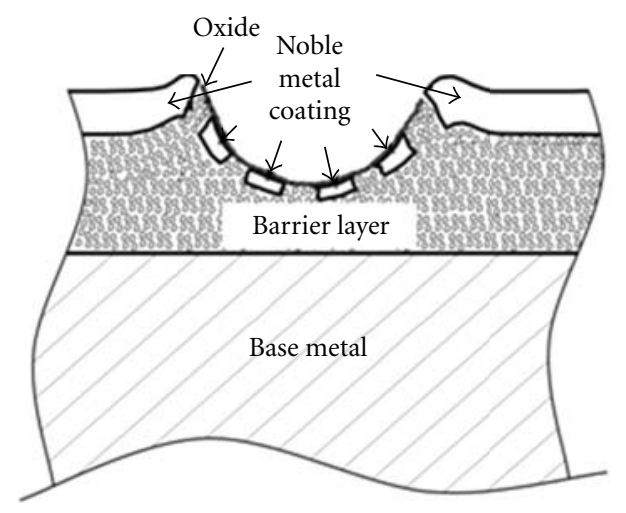

FIGURE 21: Wear pattern III partial wearing off of the upper layer and contact surface totally covered with oxide.

area and they are not covered with oxide (Figure 20). Therefore a low contact resistance is measured in this case. The electric contacts work properly.

However precious metal at the bottom of the wear crater is not the only criterion for the proper working of electric contacts. If the precious metal pieces are covered with debris of oxide (Figure 21), the contacts will also fail.

A close dependence between wear of gold and wear pattern can be observed on nanoscale particles modified gold plating. Figures 22(a) and 22(b) show the wear area of two specimens after 50,000-cycle tests. The specimen with a low electrical resistance has much lower wear, thus a lot of gold can still be observed after a 50,000-cycle test, Figure 22(a), speaks for the favorable wear pattern II. The specimen with a high contact resistance after a 50,000-cycle test has a much higher degree of wear and the contact area is completely covered with nickel of the interlayer and oxide indicated by the dark black color (Figure 22(b)). This is a typical appearance of wear pattern $\mathrm{I}$.

Wear resistance and wear pattern can be also analyzed with an energy dispersive X-ray spectroscopy (EDX). In the case of high wear resistance, the line scan of gold distribution displays a large amount of gold in the contact area after a long-term test (Figure 23(a)). In the case of poor wear resistance of gold plating, the line scan of gold distribution

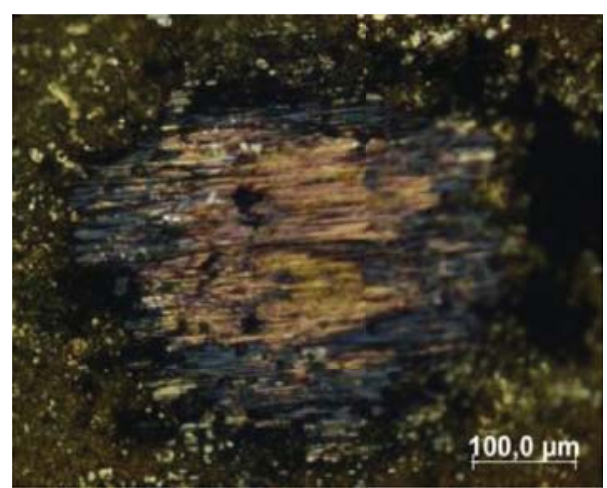

(a)

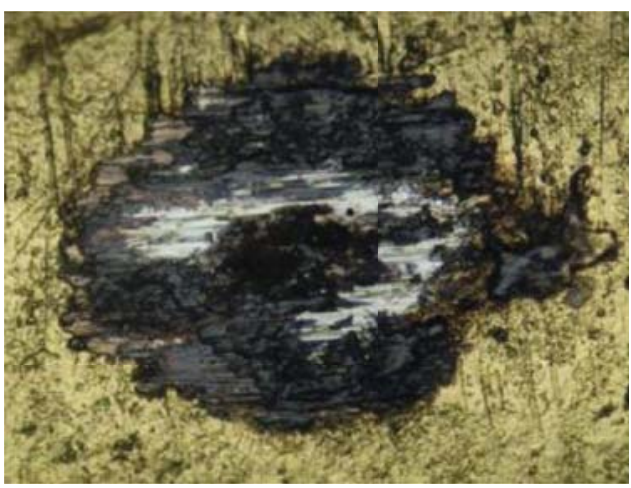

(b)

Figure 22: (a) Microscopy of contact area (average depth of wear crater: $4 \mu \mathrm{m}$, contacts survive a 50,000-cycle test, nanoscale particles modified plating of $0.8 \mu \mathrm{m}$ ). (b) Microscopy of contact area (average depth of wear crater: $6 \mu \mathrm{m}$, contacts fail at 50,000-cycle, nanoscale particles modified plating of $0.8 \mu \mathrm{m}$ ).

shows a much smaller amount of gold in the contact area after a long-term test (Figure 23(b)).

\section{Conclusion}

Our study indicates that there is a very good correlation between the rate of wear and lifetime of electrical contacts. This means that many measures used for improving bearing materials in addition to knowledge and experience gained in tribology can also be applied to coating materials of electrical contacts.

The first approaches have been conducted with the modification of the gold platings by means of hard alloying elements and nanoscale particles. They can increase the wear resistance of gold platings and therefore lead to a large increase in the lifetime of electric contacts. A combination of conditions has to be met in order to reach the desired lifetime improvement. Among these conditions, the amount, size, and combination of nanoparticles in gold platings, the thickness of both the gold platings and the nickel interlayer, and the topography of the gold platings are of most importance.

Many experiments are still required in order to find the optimal nanoscale particles for gold platings, to master the 


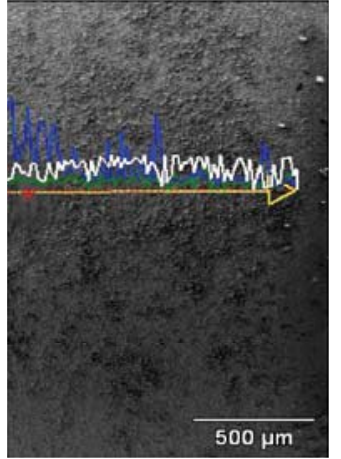

(a)

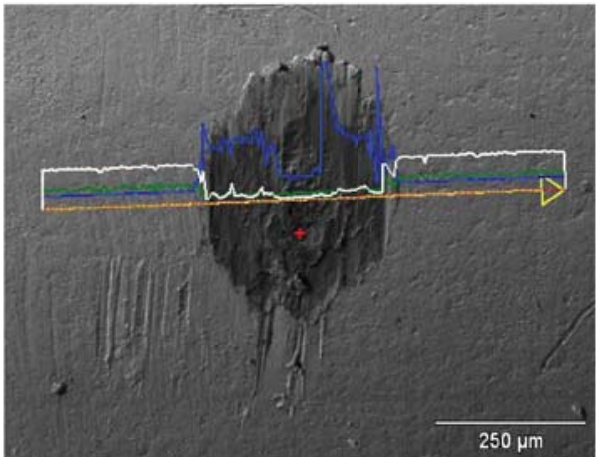

(b)

Figure 23: (a) Line scans of gold in the contact area (white line). Due to the high wear resistance of gold plating, contact survives a more than 50,000-cycle test. Average depth of wear crater was $4 \mu \mathrm{m}$. (b) Line scans of gold in the contact area (white line). Due to the poor wear resistance, the gold plating is worn out after a 50,000-cycle test. Average depth of wear crater was $6 \mu \mathrm{m}$.

electroplating process and the production of nanoscale particles. The key issues of nanoscale particles are stable particle size distribution and particle distribution in gold plating.

\section{Acknowledgments}

The investigation was financially supported by the European Union (EU, Project Nanogold) and the German State of Northrhine-Westfalia (NRW). Enthone GmbH, Langenfeld, RWTH University, Aachen, University of Paderborn, KME, Stolberg and Phoenix Contact, Blomberg supported the investigation with materials, specimens, and measurements.

\section{References}

[1] M. Braunovic, V. V. Konchits, and N. K. Myshkin, Electrical Contacts, CRC Press, Boca Raton, Fla, USA, 2007.

[2] M. Antler, "Tribology of electronic connectors," in Electrical Contacts-Principles and Applications, P. Slade, Ed., pp. 332364, Marcell Dekker, New York, NY, USA, 1999.

[3] E. Vinaricky, Elektrische Kontakte, Werkstoffe und Anwendungen, Springer, Berlin, Germany, 2002.

[4] G. L. Hornyak, H. F. Tibbals, J. Dutta, and J. J. Moore, Introduction to Nanoscience \& Nanotechnology, CRC Press, Boca Raton, Fla, USA, 2009.

[5] A. H. Slocum, Precision Machine Design, SME, Dearborn, Mich, USA, 1992.

[6] L. Wang, Analyse der Einflussparameter und Wirkungsmechanismen von Nanopartikeln in der Oberflächenschutzschicht elektrischer Kontakte, M.S. thesis, Ostwestfalen-Lippe University of Applied Sciences, Lemgo, Germany, 2011.

[7] L. Shmidt, Optimierung der Oberflächen für elektrische Kontakte, Ph.D. thesis, Ostwestfalen-Lippe University of Applied Sciences, Lemgo, Germany, 2010.

[8] J. Song and C. Koch, "Wear patterns and lifetime of electric contacts," in Proceedings of the 54th IEEE Holm Conference on Electrical Contacts (HOLM '08), pp. 238-244, October 2008. 

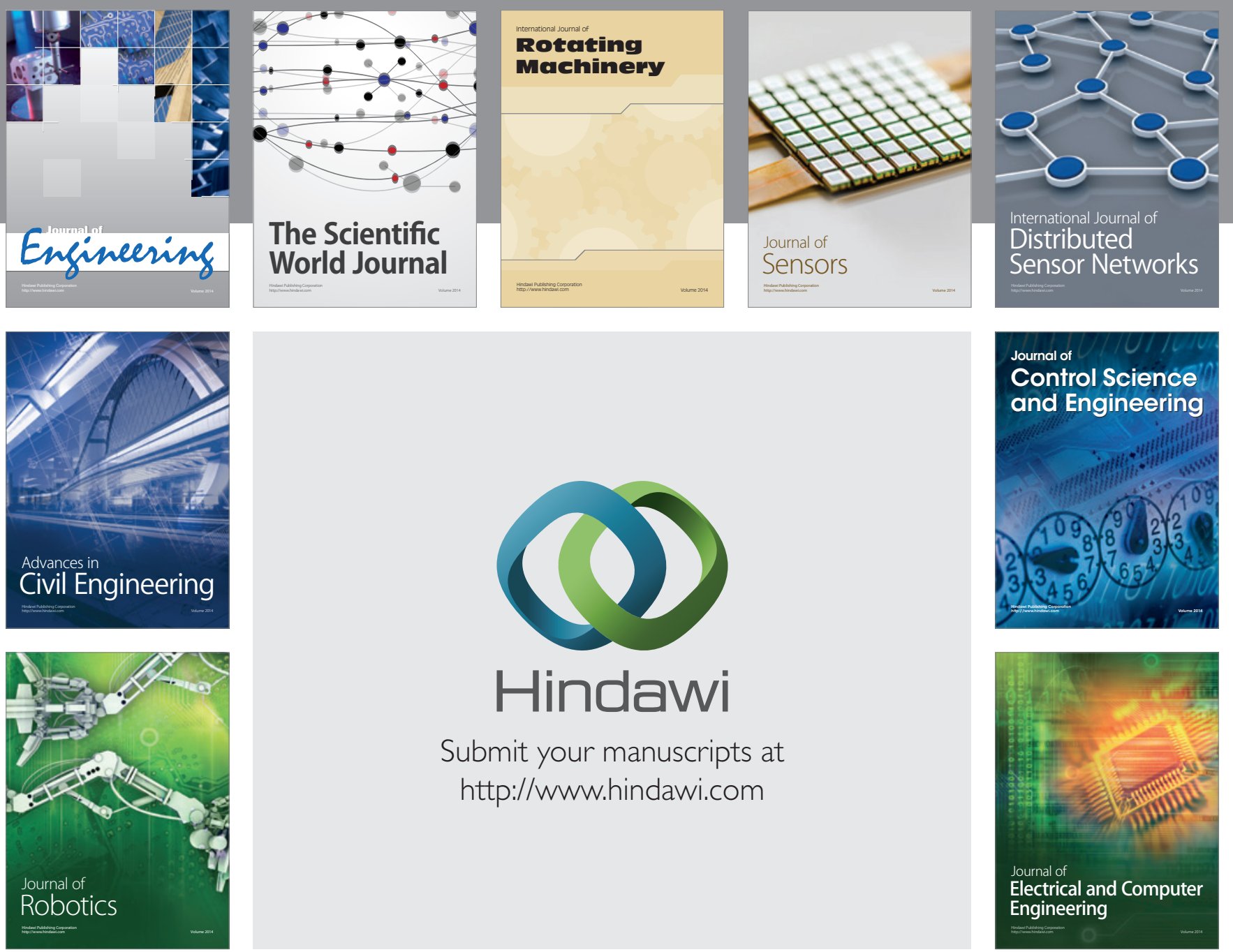

Submit your manuscripts at

http://www.hindawi.com
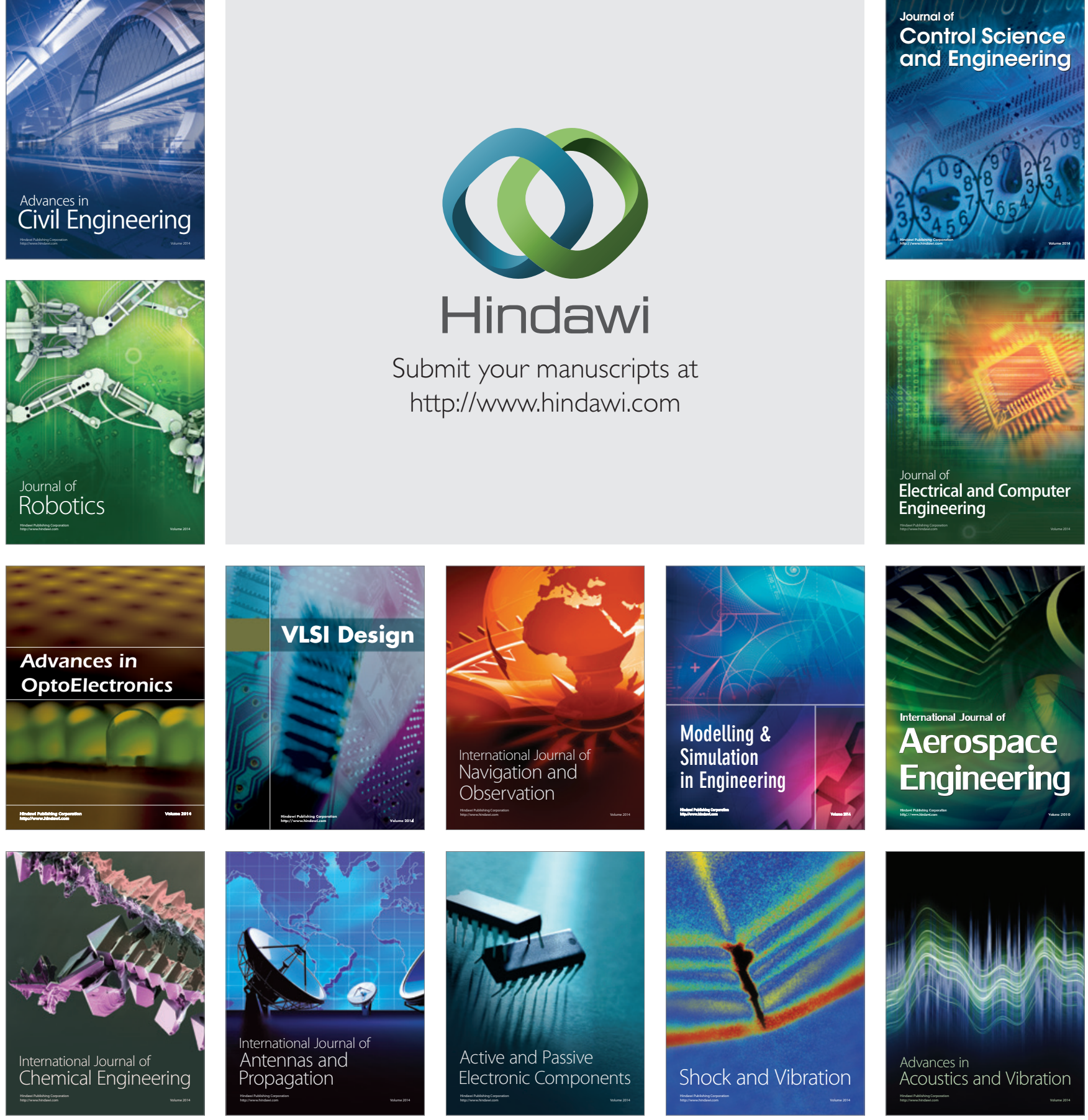